\title{
Varicella meningitis in pregnancy: a clinical conundrum
}

\author{
Baljeet K. Gholkar*, Lynda Verghese
}

Department of Obstetrics and Gynecology, Wrexham Maelor Hospital, Wrexham, Wales, United Kingdom

Received: 29 December 2019

Revised: 22 January 2020

Accepted: 29 January 2020

\section{*Correspondence:}

Dr. Baljeet K. Gholkar,

E-mail: baljeetbmc@yahoo.com

Copyright: () the author(s), publisher and licensee Medip Academy. This is an open-access article distributed under the terms of the Creative Commons Attribution Non-Commercial License, which permits unrestricted non-commercial use, distribution, and reproduction in any medium, provided the original work is properly cited.

\begin{abstract}
Authors present this experience of managing a case of Varicella meningitis in pregnancy in a patient presenting with headache in the third trimester. There was no history of dermatomal pain or rash. After intracranial hemorrhage and thrombosis were ruled out by imaging, a decision to perform a lumbar puncture was taken. The diagnosis was made following a PCR analysis on the cerebro-spinal fluid. Retrospective testing revealed immunity to varicella at booking, thus confirming reactivation of the infection. Treatment was done using intravenous acyclovir with complete recovery. It also created a clinical dilemma for the best possible monitoring plan for the fetus to rule out affection. A fetal medicine scan revealed no structural defects in the fetus. The subsequent pregnancy period was uneventful. A well grown normal baby was born at term. This case highlights the significance of considering a lumbar puncture in cases of intractable headache and also highlights the dilemma for fetal monitoring in such rare presentations.
\end{abstract}

Keywords: Fetal varicella, Headache in pregnancy, Varicella meningitis

\section{INTRODUCTION}

Varicella meningitis is a rare complication of varicella zoster virus (VZV) infection. Most cases that have been reported are in immunocompromised or frail elderly patients. ${ }^{1,2}$ This case is a first description of the complication in an otherwise fit and well pregnant patient possibly secondary to immuno-suppression of pregnancy. It was an uncharacteristic presentation of the condition with no prodromal rash or dermatomal pain. The diagnosis was not suspected clinically and only reached after the lumbar puncture. Early consideration of lumbar puncture helped reach the diagnosis and initiate prompt treatment.

\section{CASE REPORT}

A 27-year-old primi-gravida presented to the maternal assessment unit of this hospital with an acute onset headache at 32-week gestation. The headache was constant and throbbing in character with a sudden increase in severity on the day prior to presentation. She described it as the worst headache of her life. It was accompanied by photophobia and one episode of vomiting. She had a history of having a flu like illness a few days ago but no history of rash.

This was a spontaneously conceived pregnancy. In the past medical history, she was a known epileptic on lamotrigine. She was seizure free for 9 years and under regular medical follow up. She had a history of a recent dental abscess which was treated with oral antibiotics for 7 days. She was a heavy smoker but was motivated and working towards reducing/quitting smoking.

On examination, she was conscious and alert with a GC score of 15 . The neurological examination revealed no focal defects or any signs of meningism. Fundoscopy was normal as well. Her booking BMI was 26. The initial clinical impression was a possible sub-arachnoid hemorrhage or a cavernous sinus venous thrombosis. 


\section{Investigations}

She had an MR imaging and venogram of the brain which did not show any intracranial haemorrhage or a thrombus in the venous sinuses. The orbits and para-nasal sinuses were normal as well. After the imaging, based on a medical opinion, decision was made to offer a lumbar puncture which was accepted. The results of the lumbar puncture are shown in Table 1.

Table 1: Results of lumbar puncture.

\begin{tabular}{|ll|}
\hline Parameter & Value \\
\hline Colour & Colourless \\
\hline Pressure $\left(\mathrm{cm} \mathrm{H}_{2} \mathrm{O}\right)$ & Normal \\
\hline WBC COUNT $(\mathrm{X}$ & $365(100 \%$ Lymphocytes, $0 \%$ \\
$\left.10^{6} / \mathrm{L}\right)$ & Polymorphs $)$ \\
\hline Glucose $(\mathrm{mmol} / \mathrm{L})$ & Normal \\
\hline Protein $(\mathrm{gm} / \mathrm{L})$ & 1 \\
\hline Gram stain & Negative \\
\hline WBC: white blood cell. & \\
\hline
\end{tabular}

The result was suggestive of a possible viral meningitis. The CSF was further analyzed using PCR for specific viruses (HSV type 1 and 2, Enterovirus, Varicella Zoster, Meningococcus and Pneumococcus) which came back as positive for Varicella Zoster.

\section{Treatment}

Treatment with intravenous acyclovir was immediately commenced for 2 weeks with a plan for a repeat lumbar puncture after treatment.

A retrospective enquiry after this result revealed that she had a definite history of chicken pox as a child. A varicella zoster IgG analysis was done on the booking bloods which was positive as well. This confirmed that this was possibly a reactivation of the virus. HIV testing which was declined initially by the patient was accepted after this diagnosis and was negative.

\section{Outcome and follow up}

A repeat lumbar puncture after the treatment was negative for PCR of VZV. Symptomatically there was considerable improvement after commencing treatment. After a multi-disciplinary discussion regarding this case, it was decided to consider this episode of infection as an active infection possibly secondary to reactivation of the virus. Knowing the risks of an active varicella infection, a fetal medicine referral was done after 4 weeks of the diagnosis. The fetal medicine scan revealed no abnormality.

Thereafter she had an uneventful course of pregnancy and had an emergency cesarean section following a failed induction of labour for maternal epilepsy at 38 weeks. A neonatal survey on the child was normal and the baby did not require any neonatal special care.

\section{DISCUSSION}

Most of the reported cases of varicella meningitis have been in immunocompromised or frail elderly patients. ${ }^{1,2}$ Recently, there have been few reports of the condition in immunocompetent individuals with prior history of varicella infection. ${ }^{3}$ To the best of this knowledge, this is the first reported case of varicella meningitis in an otherwise immuno-competent pregnant female. The prior reported case in pregnancy has been in a woman with AIDS. ${ }^{4}$

Authors believe the overall number of reported cases in the past has been low due to a possible under diagnosis of the condition as it can present with variable and mild symptoms. This case also was diagnosed only after the PCR test was done on the CSF and clinically there were no suggestive symptoms. Based on this case experience, authors recommend an early consideration of lumbar puncture in cases of intractable headache. Authors also want to highlight the importance of CNS imaging prior to planning a lumbar puncture.

There have been case reports with late onset of rash occurring 7-21 days after neurological symptoms. ${ }^{5}$ This is possibly because the pathogenesis of this secondary infection involves the reactivation of viral particles in the ganglion with a relatively low viral load. The initial manifestation can be with CNS symptoms and then can have a late rash or dermatomal pain and can also lead to a severe CNS infection if treatment is not commenced early. In this case, there was a quick response to antiviral medication and possibly due to the resolution of the infection, there were no late symptoms.

RCOG guideline on chicken pox in pregnancy mentions of a possible risk of transmission of the infection across the placenta to the fetus in a woman who has a primary infection in pregnancy. This risk as stated in the guideline is still low at less than 1 percent. $^{6}$ This chance of fetal infection is secondary to no transferable immunity from the mother to the fetus. In a case of secondary infection, this potential risk should be minimal or low as there will be transferable IgG antibodies in the mother. This case had immunity to varicella at booking. This case was discussed in a multi-disciplinary team meeting consisting of neonatologists, microbiologists, obstetric and internal medicine physicians. The final consensus was that due to the overall lack of data regarding the risks of secondary infection on the fetus and the severity of the presentation, fetal monitoring with scans and a neonatal survey was recommended. This part of this management plan is debatable however multidisciplinary opinions should be sought on and individualized plans should be made in difficult cases such as this one. 


\section{Learning points from this case are as follows}

- The acute presentation of headache and photophobia was possibly suggestive of an intracranial cause but the imaging did not reveal a bleed or hemorrhage. Authors recommend early consideration of lumbar puncture in diagnostic dilemmas such as this case. This avoids any delays in initiating treatment. Raised intracranial pressure however has to be ruled out prior to going ahead with lumbar puncture.

- This case highlights how PCR has revolutionized diagnosis of VZV meningitis in patients with no specific symptoms.

- A detailed history and assessment to rule out other causes of immune-suppression (HIV, steroids) should be done in cases of viral meningitis. This case was most likely secondary to pregnancy led immunesuppression.

- Viral meningitis can have late manifestation of skin rash or dermatomal pain and also may not have any known symptoms as in this case.

The role of fetal monitoring in this case was considered due to the lack of data on the potential for active secondary infections causing fetal affection. Any such episode.

Funding: No funding sources Conflict of interest: None declared

Ethical approval: Not required

\section{REFERENCES}

1. Takayama N, Yamada H, Kaku H. Herpes zoster in immunocompetent and immunocompromised Japanese children. Pediatr Int. 2000;42:275-9.

2. Ellis D, Barsell A, Riahi R, Stumpf B. Varicella zoster virus encephalitis in a patient with disseminated herpes zoster: report and review of the literature. Dermatol Online J. 2014;21(3).

3. Kangath RV, Lindeman TE, Brust K. Herpes zoster as a cause of viral meningitis in immunocompetent patients. Case Reports. 2013;2013:bcr2012007575.

4. Jayakrishanan A, Vrees R, Anderson B. Varicella zoster meningitis in a pregnant woman with acquired immunodeficiency syndrome. Am J Perinatol. 2008;25(9):573-5.

5. Sanguankeo A, Upala S, Sornprom S, Thamcharoen N. Varicella-zoster meningitis with a late-onset of skin eruption. BMJ Case Rep. 2015;2015:bcr-2014208056.

6. Royal College of Obstetricians and Gynaecologists. Chickenpox in Pregnancy. Green-top Guideline No. 13. London: RCOG; 2015:1-17.

Cite this article as: Gholkar BK, Verghese L. Varicella meningitis in pregnancy: a clinical conundrum. Int J Reprod Contracept Obstet Gynecol 2020;9:1300-2. 